\title{
A PROSPECTIVE STUDY OF NON-SPECIFIC ABDOMINAL PAIN IN OUR INSTITUTE
}

\author{
Suresh Sambandam¹, Raghupathy², Arikrishnan Vaithiswaran³, Palanisamy4, Prasanth ${ }^{5}$
}

1 Professor, Department of General Surgery, Chengalpattu Medical College and Hospital, Chengalpattu. 2 Professor, Department of General Surgery, Chengalpattu Medical College and Hospital, Chengalpattu. 3Junior Resident, Department of General Surgery, Chengalpattu Medical College and Hospital, Chengalpattu. 4Junior Resident, Department of General Surgery, Chengalpattu Medical College and Hospital, Chengalpattu. 5Junior Resident, Department of General Surgery, Chengalpattu Medical College and Hospital, Chengalpattu.

\section{ABSTRACT}

\section{BACKGROUND}

While in general practice chronic non-specific abdominal complaints are common; there is insufficient data on the clinical course and the management of these complaints. Aim of this study was to present a primary care based profile of these chronic complaints including health care involvement, health status and clinical course.[1],[2]

\section{METHODS}

A prospective study with using feasible materials.

\section{RESULT}

Of 146 cases studied with NSAP, most were females and most had chronic abdominal pain and needs other acute abdomen condition to be excluded prior.

\section{CONCLUSION}

It is not a safe diagnosis to be considered at the initial stage of admission, but is only a diagnosis by exclusion.

\section{KEYWORDS}

Abdominal Pain, Complications, Diagnosis, Therapy, Adolescent, Adult, Aged, Female, Human, India, Male, Middle Age.

HOW TO CITE THIS ARTICLE: Sambandam S, Raghupathy, Vaithiswaran A, et al. A prospective study of non-specific abdominal pain in our institute. J. Evolution Med. Dent. Sci. 2016;5(71):5214-5217, DOI: 10.14260/jemds/2016/1181

\section{INTRODUCTION}

Five to ten percent of all admissions to the Emergency Department (ED) are due to acute abdominal pain. Acute Nonspecific Abdominal Pain (NSAP) is defined as acute abdominal pain less than one week in duration, for which there is no diagnosis despite investigations and comprises a spectrum of undiagnosed conditions, both somatic and functional and remains a "diagnosis of exclusion."

The necessity to achieve a correct diagnosis and a systematic approach to NSAP should be useful in order to reduce the admission rate for NSAP because of the costs and morbidity associated with this condition in terms of excessive hospital stay, multiple investigations and unnecessary surgical explorations.[3-6] Systematic baseline characters examination, abdominal Ultrasound (US) abdominal Computed Tomography (CT), and Early Laparoscopy (EL) have all been described as potential methods for improving the diagnostic accuracy in the emergency setting.

In conclusion, the management of acute NSAP can be divided into three stages. The first stage is the initial history, physical examination, baseline investigations and formulation of a working and differential diagnosis.

Financial or Other, Competing Interest: None.

Submission 28-07-2016, Peer Review 21-08-2016,

Acceptance 27-08-2016, Published 03-09-2016.

Corresponding Author:

Dr. Suresh Sambandam,

Professor,

Department of General Surgery,

Chengalpattu Medical College \& Hospital,

Chengalpattu.

E-mail: suresam10@gmail.com

DOI: $10.14260 /$ jemds/2016/1181
The second stage involves the judicious use of radiologic techniques. The third stage should be represented by diagnostic laparoscopy. Enhanced or non-enhanced computed tomography of the abdomen and pelvis is a crucial step after baseline examination and before invasive diagnostic methods such as diagnostic laparoscopy.

Diagnostic laparoscopy is helpful in treatment of patients with NSAP, because it improves the achievement of correct diagnosis and reduces hospital stay and per patient costs, although seems to be unuseful in prevention of recurrence of symptoms.

The results of our systematic review suggest that there is insufficient evidence at present to justify routine use of early laparoscopy in patients with undifferentiated acute abdominal pain. For this reason, laparoscopy should be reserved to patients with worsening course or when urgency is reasonably suspected after baseline. ${ }^{[7-10]}$

- A disorder characterised by a sensation of marked discomfort in the abdominal region.

- Painful sensation in the abdominal region.

- Sensation of discomfort, distress or agony in the abdominal region; generally associated with functional disorders, tissue injuries or diseases.[11-15]

- Your abdomen extends from below your chest to your groin. Some people call it the stomach, but your abdomen contains many other important organs. Pain in the abdomen can come from any one of them. The pain may start somewhere else, such as your chest. Severe pain does not always mean a serious problem nor does mild pain mean a problem is not serious. Call your healthcare provider if mild pain lasts a week or more or if you have pain with other symptoms. 


\section{AIM OF THE STUDY}

Nonspecific abdominal pain constitutes a considerable proportion of surgical admissions with important implication for bed occupancy, manpower provisions and cost to health service and above all decision making in management of the patients with acute abdomen and chronic abdomen pain.

- This study aims at defining this entity.

- Defining the condition, its incidence and natural history.

- Pathway of evaluation of these patients.

- Assesses the usefulness of relevant investigations to rule out organic causes.

- Is it a safe diagnosis?

- To prove that NSAP forms a considerable part of hospital admissions without a knowledge of which patient management leads to wastage of resources and more than that avoiding unnecessary surgery.[16-19]

\section{MATERIALS AND METHODS}

NSAP is as condition with no specific explanatory disease or cause. A study of 146 pts. admitted at CMCH between 2014 and 2015 with acute abdominal pain was conducted. NSAP being the beginning condition has low morbidity and identifying these cases is of importance in avoiding unnecessary surgery.

Most of the patients in the study were admitted as acute abdominal pain and as emergency from the casualty department. Patients were studied at the surgical wards or from medical wards where surgeon's opinion were sought.

A through history taking and physical examination will yield a diagnosis many times, but since NSAP mimics an acute abdomen no chance are taken and some supportive evidence from investigations should be present to come to the diagnosis of NSAP. The model questionnaire proforma used for obtaining the history and recording the physical signs is filed in the annexure page.[9],[20-23]

\section{All the Patients had the Preliminary Investigations done at Admission. They were,}

- Haemogram.

- Blood sugar.

- Urea.

- Creatinine.

- $\quad$ ECG.

- X-ray chest and erect abdomen.

With resuscitative measures done simultaneously, abdominal sonogram was done in many of the patients. Being an easy diagnostic approach and a technique constantly accurate for upper abdomen and pelvis and its availability in GH around the clock and easily available, USG was made use of. In a few cases where most of the tests were not contributing a clue to diagnosis had diagnostic laparoscopy and eight patients underwent exploratory laparotomy. Analysis with regard to male-to-female ratio, type of presentation, age, incidence, associated symptoms and signs, the investigations done and management of patients and outcome is discussed.

\section{RESULTS OF THE STUDY}

\section{Sex Incidence}

Male 58 (39.7\%):female $88(60.3 \%)$, there was not only female predominance in the overall ratio but also in each group. The ratio varies between 0.4 in above 40 years' age group and 0.8 in 20-40 years' age group. This confirms to the already established literature data of female preponderance. Obvious female preponderance is made out with male:female ratio of $2: 3$. Abdominal pain not confirming to any particular diagnosis after investigations were found to have similar exaggerated female distribution to the often complained "generalised body ache." Psychogenic factors, low pain threshold, less stress tolerance may be contributing factors.

\section{Age Distribution}

About $60 \%$ of the cases are seen in $3^{\text {rd }}$ to $5^{\text {th }}$ decade of age group, both in males and females. Such a preponderance in 20 40 years' age group may be attributed to the stress factor contributing to psychogenic aetiology.

\section{Type of Presentation}

109 pts. present as acute abdominal pain with 68 of them female and 37 presented as chronic with 24 of them female. Abdominal pain present for more than 2 weeks after or before investigation causing repeated hospital visits after or before investigations were considered chronic. Pain for less than 2 weeks was almost thrice as common as chronic, the reason probably is extensive investigations reveal at least a positive diagnostic test leading to the pain being categorised under that diagnosis and being eliminated from the non-specific category. The chronicity of pain also leads to an extensive work-up for psychogenic aetiology and the required therapy.

\section{Symptoms}

Abdominal pain which was diffuse in 76, of them 54 were female. Upper abdominal pain for 38, of them 13 were female. Lower abdominal pain in 32 patients, of them 21 were female. Loss of appetite in 50 pts., myalgia in 42 patients, dysuria in 28 patients, dyspepsia in 24 patients, nausea and vomiting in 12 patients.

\section{Signs}

Only 34 cases presented with tenderness and in remaining 112 cases it were absent. Most of the patients were clinically suspected to have acid peptic disease (40 cases) with no evidence of pathology on endoscopy and ultrasound of abdomen, was more common in males. Pelvic inflammatory disease were suspected in about 21 out of 32 female cases complaining of lower abdominal pain and dysuria/leucorrhoea remains unproved. Only $10 \%$ of patients with tuberculosis abdomen fell into the NSAP category for response to empirical, anti-tuberculous therapy also formed part of diagnostic criteria for Koch's abdomen. According to clinical diagnosis made, investigations were opted for. The 146 cases study had all the relevant tests negative. Among the investigations, USG was the most commonly used one, though was followed with further relevant investigations. Laparoscopy and biopsy was done in cases suspected to have abdominal tuberculosis and some were put on antituberculous chemotherapy with negative results, both were categorised to NSAP. Few went for negative laparotomy too.

\section{DISCUSSION}

Nonspecific Abdominal Pain (NSAP) is not a defined disease, but is a collection of conditions. The term NSAP refers to an abdominal or pelvic pain of less than 7 days' duration for which the diagnosis remains uncertain after clinical examination and baseline investigations. 
NSAP is a significant problem in general surgery and accounts for an estimated $13 \%$ to $40 \%$ of emergency surgical admissions for acute abdominal pain. The mean hospital stay for patients admitted with NSAP ranges between 4 and 6 days using the traditional "wait and see" management. This includes repeated clinical examination, radiologic investigation and a gynaecologic opinion. A delay in surgical intervention while further investigations are performed may increase morbidity and prolong hospital stay. The end result may be an unsatisfactory discharge from hospital after a prolonged stay with a high percentage of patients leaving the hospital without a precise clinical diagnosis.

Many suggestions have been made about improving diagnostic rates in acute NSAP. DeDombal et al ${ }^{17}$ have suggested that the proportion of correct diagnoses can be greatly increased by the use of structured questionnaires and diagnostic programs on computers. Although computer-aided diagnosis can improve diagnostic rates by at least $20 \%$, these programs are unpopular. Other researchers have suggested ultrasonography, thermography, peritoneal cytology, CT and more recently spiral computer tomography. Nevertheless, none of these techniques seems to have been determinant in improving the clinical management of NSAP.

In the management of acute abdomen laparoscopy has both a diagnostic and a therapeutic role. The use of diagnostic laparoscopy in patients with acute abdominal pain is not new and many studies have demonstrated an improvement in surgical decision making associated with its use, particularly when the need of operation is uncertain. Therefore, NSAP appears to be a good indication to early laparoscopy to improve diagnostic rates and reduce hospital stay and costs.

In the 1990s, 2 RCTs compared early laparoscopy to active clinical observation. Both trials showed that early laparoscopy clearly facilitated the establishment of a diagnosis with subsequent therapy ( $97 \%$ and $81 \%$ after early laparoscopy vs. $28 \%$ and $36 \%$ after clinical observation), whereas more patients in the control group left the hospital without a clear diagnosis. Furthermore, hospital stay was shorter in the laparoscopic group in both trials, while morbidity and mortality were similar. Although these trials had comparable results, their design presented in our opinion some limits. The study by Champault et al did not include abdominal ultrasound among the baseline examinations, presented results limited to the perioperative period and was limited in its statistical power due to a small number of patients (30 in each group). We think that nowadays abdominal ultrasound should be part of the baseline investigations of an acute abdomen, and that a minimum of 12 months of follow-up is essential to evaluate NSAP recurrence rate. Furthermore, while NSAP literature is focused exclusively on right iliac fossa pain in young women, the study by Decadt et al included men and women with acute abdominal pain regardless of pain localisation.

\section{CONCLUSION}

From the study of 146 cases of NSAP between 2014 and 2015, the following conclusion is arrived at; $25-35 \%$ of cases on general surgery outpatient present with abdominal pain. Of them $70-80 \%$ of patients were treated as per their symptoms. NSAP accounts for one-third of those cases. Patients admitted to hospital with acute NSAP represent only a tip of the iceberg of the problem. In the absence of an organic cause in NSAP, the possibility of pain of psychogenic origin should be considered.
Chronic abdominal pain is more often psychological than a surgical problem. It is good to consider operative entities before non-operative processes in the differential diagnosis of abdominal pain. A prudent surgeon considers even if only briefly all the possibilities before proceeding to operation. It is thus not a safe diagnosis to be considered at the initial stage of admission, but is only a diagnosis by exclusion.

\section{REFERENCES}

1. Sheridan WG, White AT, Havard T, et al. Non-specific abdominal pain: the resource implications. Ann R Coll Surg Engl 1992;74(3):181-5.

2. Paterson-Brown S. Emergency laparoscopy surgery. Br J Surg 1993;80(3):279-83.

3. Raheja SK, McDonald PJ, Taylor I. Non-specific abdominal pain, an expensive mystery. J R Soc Med 1990;83(1):1011.

4. Hawthorn IE. Abdominal pain as a cause of acute admission to hospital. J R Coll Surg Edinb 1992;37(6):389-93.

5. Sellors J, Mahony J, Goldsmith C, et al. The accuracy of clinical findings and laparoscopy in pelvic inflammatory disease. Am J Obstet Gynecol 1991;164(1 Pt 1):113-20.

6. Brown DL, Doulbilet PM. Transvaginal sonography for diagnosing ectopic pregnancy: positive criteria and performance characteristics. J Ultrasound Med 1994;13(4):259-66.

7. Olsen JB, Myrén CJ, Haahr PE. Randomized study of the value of laparoscopy before appendectomy. Br J Surg 1993;80(7):922-3.

8. Adams ID, Chan M, Clifford PC, et al. Computer-aided diagnosis of acute abdominal pain: a multicentre study. BMJ 1986;293(6550):800-4.

9. Allemann F, Cassina P, Rothlin $\mathrm{M}$, et al. Ultrasound scans done by surgeons for patient with acute abdominal pain: a prospective study. Eur J Surg 1999;165(10):966-70.

10. Rao PM, Rhea JT, Novelline RA, et al. Effect of computed tomography of the appendix on treatment of patients and use of hospital resources. N Engl J Med 1998;338(3): 141-6.

11. Champault G, Rizk N, Lauroy J, et al. Right iliac fossa in women: conventional diagnostic approach versus primary laparoscopy. A controlled study (65 cases). Ann Chir 1993;47(4):316-9.

12. Decadt B, Sussman L, Lewis MP, et al. Randomized clinical trial of early laparoscopy in the management of acute nonspecific abdominal pain. Br J Surg 1999;86(11):1383-6.

13. Hager WD, Eschenbach DA, Spence MR, et al. Criteria for diagnosis and grading of salpingitis. Obstet Gynecol 1983;61(1):113-4.

14. Grunewald B, Keating J. Should the normal appendix be removed at operation for appendicitis? J R Coll Surg Edinb 1993;38(3):158-60.

15. Carr NJ. The pathology of acute appendicitis. Ann Diagn Pathol 2000;4(1):46-58.

16. Irvin TT. Abdominal pain: a surgical audit of 1190 emergency admissions. Br J Surg 1989;76(11):1121-5.

17. De Dombal FT, Leaper DJ, Horrocks JC, et al. Human and computer-aided diagnosis of abdominal pain: further report with emphasis on performance of clinicians. BMJ 1974;1(5904):376-80.

18. Sutton GC. Computer-aided diagnosis: a review. Br J Surg 1989;76(1):82-5. 
19. Sugarbaker PH, Bloom BS, Sanders JH, et al. Preoperative laparoscopy in diagnosis of acute abdominal pain. Lancet 1975;1(7904):442-5.

20. Greason KL, Rappold JF, Liberman MA. Incidental laparoscopic appendectomy for acute right lower quadrant abdominal pain. Surg Endosc 1998;12(3): 223-5.
21. Gaitàn H, Angel E, Sànchez J, et al. Laparoscopic diagnosis of acute lower abdominal pain in women of reproductive age. Int J Gynecol Obstet 2002;76(2):149-58.

22. Poulin EC, Schlachta CM, Mamazza J. Early laparoscopy to help diagnose acute non-specific abdominal pain. Lancet 2000;355(9207):861-3.

23. Gupta H, Dupuy DE. Advances in imaging of the acute abdomen. Surg Clin North Am 1997;77(6):1245-63. 